\title{
Making soccer kicks better: A Study in Particle Swarm Optimization and Evolution Strategies
}

\author{
Namrata Khemka \\ Dept. of Computer Science \\ University of Calgary \\ Calgary, Canada \\ khemka@cpsc.ucalgary.ca
}

\author{
Christian Jacob \\ Dept. of Computer Science \\ University of Calgary \\ Calgary, Canada \\ jacob@cpsc.ucalgary.ca
}

\author{
Gerald Cole \\ Faculties of Kinesiology/Engineering \\ University of Calgary \\ Calgary, Canada \\ cole@kin.ucalgary.ca
}

\begin{abstract}
Biomechanics is a science of examining the internal and external forces on the human body. In biomechanics, forward dynamics simulation models can be used to study optimal control of the human musculoskeletal system. An example application is the soccer kick, where the optimal recruitment pattern of the muscles of the lower extremity can result in a high speed shot on net. We used evolutionary algorithmsparticle swarm optimizers and evolution strategies- to adjust muscle control parameters for a soccer kick. In this paper we describe our implementation of the soccer kick project, optimization experiments performed with the soccer kick and comparing the results from Particle Swarm Optimization and Evolution Strategies.
\end{abstract}

\section{Introduction}

Forward dynamics simulation models can be used to study optimal control of the human musculoskeletal system. This type of study lends itself to the design of equipment for sports activities. In this context, we investigate the leg and foot movements for kicking a soccer ball [5][6][7]. One of the goals of a soccer kick when shooting on net is to kick the ball as fast as possible. Controlling the overall leg and foot movements-which involve 17 muscles-requires a large number of parameters for the biomechanics simulation. Currently, it is not clear what the 'optimal' settings for the muscle control parameters are, within all physical and physiological constraints. In fact, this turns out to be a difficult optimization problem.

The movement of the lower extremity is over-determined by the musculature. Numerical optimization algorithms are typically used to estimate a solution to a posed optimal control problem, in this case maximizing forward ball speed. For example, for different distributions of mass in a soccer shoe, the stimulation patterns of the muscles of the kicking limb can be numerically optimized such that the highest velocity of the ball is obtained. To make things even more challenging, we also ask the question of how to identify global maxima when comparing different shoes. The determination of optimized settings is a prime challenge, as the simulation model consists of 56 parameters (see Section 2 for details). As the fitness evaluation of each parameter vector is time consuming (5-6 seconds for a single run, each over thousands of iterations), we strive to minimize the search time of the optimizer.

The focus of our study is to compare the performance of two evolutionary optimization techniques: Particle Swarm Optimization (PSO) [12] and Evolution Strategies (ES) [13]. This entails the visualization, analysis and interpretation of results, which will allow us to determine which of the two algorithms is most fit to find a set of optimized model parameters, or whether a different approach is required.

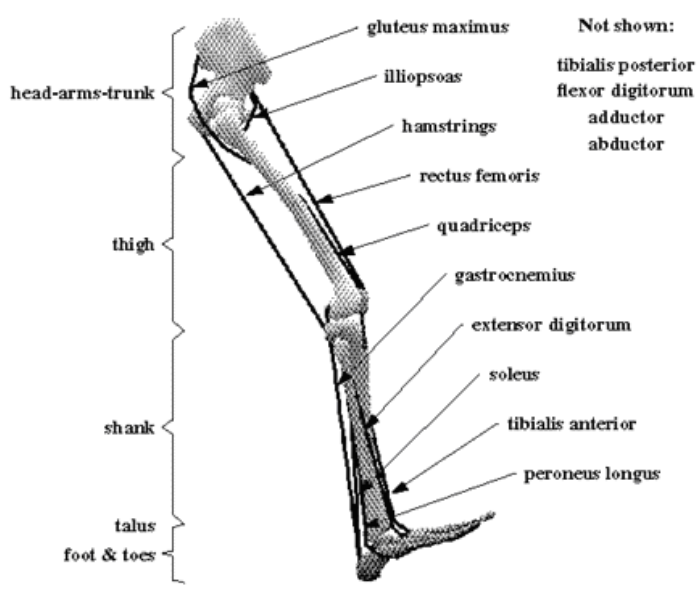

Figure 1: Muscle groups in the human leg [17].

\section{The Soccer Kick Project}

Soccer is one of the most popular sports in the world. A variety of skills regarding leg and foot movements is required since the arms and hands can not touch the ball. Kicking is one of the important techniques for playing soccer. When shooting on net, the kick can be evaluated by speed and accuracy of the ball. The musculoskeletal model used to simulate the soccer kick has been simplified to include 17 different muscle groups in the leg (Table 1), which are in the foot and toes, talus, shank, thigh and head-arms-trunk sections (Figure 1).

The biomechanical model used in this study was first proposed in [17], and extended to the soccer kick by Cole and Gerritsen [5][6]. In this model, each muscle has a start and end time for its stimulation. As seen in Figure 2, we define $t_{0}\left(M_{k}\right)$ to be the start time and $t_{\text {end }}\left(M_{k}\right)$ the end time of the stimulation for muscle $M_{k}$, where $k=1,2,3, \ldots, 17$. Each muscle is allowed to change only once during its stimulation at time $t_{c}\left(M_{k}\right)$ with, $t_{0} \leq t_{c} \leq t_{\text {end }}$. In Figure 2, $s_{1}\left(M_{k}\right)$ is the initial stimulation level, whereas the muscle 


\begin{tabular}{|l|l|l|}
\hline \multicolumn{1}{|c|}{ Muscle } & \multicolumn{1}{|c|}{ Name } & \multicolumn{1}{|c|}{ Functionality } \\
\hline \hline$M_{1}$ & Gluteus Maximus & Hip extension \\
\hline \hline$M_{2}$ & Hamstrings & $\begin{array}{l}\text { Hip extension, } \\
\text { knee flex }\end{array}$ \\
\hline \hline$M_{3}$ & Illiopsoas & Hip flex \\
\hline \hline$M_{4}$ & Rectus Fermoris & $\begin{array}{l}\text { Hip flex, knee ex- } \\
\text { tension }\end{array}$ \\
\hline \hline$M_{5}$ & $\begin{array}{l}\text { Vastus Inter- } \\
\text { medius }\end{array}$ & Knee extension \\
\hline \hline$M_{6}$ & Gastrocnemius & $\begin{array}{l}\text { Plantar flex, knee } \\
\text { flex }\end{array}$ \\
\hline \hline$M_{7}$ & Soleus & Plantar flex \\
\hline \hline$M_{8}$ & Tibialis Posterior & Inversion \\
\hline \hline$M_{9}$ & Flexor Digitorum & Plantar flex \\
\hline \hline$M_{10}$ & Tibialis Anterior & Dorsiflexion \\
\hline \hline$M_{11}$ & Peroneals & Eversion \\
\hline \hline$M_{12}$ & $\begin{array}{l}\text { Extensor Digito- } \\
\text { rum }\end{array}$ & Dorsiflexion \\
\hline \hline$M_{13}$ & Abductor & Hip abduction \\
\hline \hline$M_{14}$ & Adductor & Hip extension \\
\hline \hline$M_{15}$ & Vastus Medialis & Knee extension \\
\hline \hline$M_{16}$ & Vastus Lateralis & Knee extension \\
\hline \hline$M_{17}$ & Castus Oblique & $\begin{array}{l}\text { Hip flex, knee ex- } \\
\text { tension }\end{array}$ \\
\hline
\end{tabular}

Table 1: Functionality of each of the 17 muscles represented in our model. Eversion is defined as turning the foot out away from the medial (midline of the body), whereas inversion is defined as turning the foot inward towards the medial. Dorsiflexion is the bending of the foot upwards. A plantar flex is the toe-down motion (flexing) of the foot at the ankle.

stimulation changes at time $t_{c}\left(M_{k}\right)$ to $s_{2}\left(M_{k}\right)$. Hence, the control parameter vector for muscle $M_{k}$ is represented as:

$$
\operatorname{ctrl}_{M_{k}}=\left(s_{1}\left(M_{k}\right), s_{2}\left(M_{k}\right), t_{0}\left(M_{k}\right), t_{c}\left(M_{k}\right), t_{\text {end }}\left(M_{k}\right)\right) \text {. }
$$

For all our experiments we set $t_{0}\left(M_{k}\right)=0, t_{\text {end }}\left(M_{k}\right)=$ 1 and $k=1, \ldots, 9,11, \ldots, 17$. When the leg is raised backwards for kicking, muscle $M_{10}$ prevents the shin from hyper-extending. Right before the toe comes in contact with the ball, $M_{10}$ stiffens up the ankle joint. As a result, the stimulation of this muscle changes twice during a simulation and is represented by:

$$
\begin{gathered}
\operatorname{ctrl}_{M_{10}}=\left(s_{1}\left(M_{10}\right), s_{2}\left(M_{10}\right), s_{3}\left(M_{10}\right),\right. \\
\left.t_{0}\left(M_{10}\right), t_{c_{1}}\left(M_{10}\right), t_{c_{2}}\left(M_{10}\right), t_{\text {end }}\left(M_{10}\right)\right),
\end{gathered}
$$

where $s_{1}\left(M_{10}\right)$ is the initial stimulation level, the first muscle stimulation changes at time $t_{c_{1}}\left(M_{10}\right)$ to $s_{2}\left(M_{10}\right)$ and the second stimulation changes at time $t_{c_{2}}\left(M_{10}\right)$ to $s_{3}\left(M_{10}\right)$.

The parameters of the 17 muscles together with the coordinates of the ball position result in a 56-dimensional search

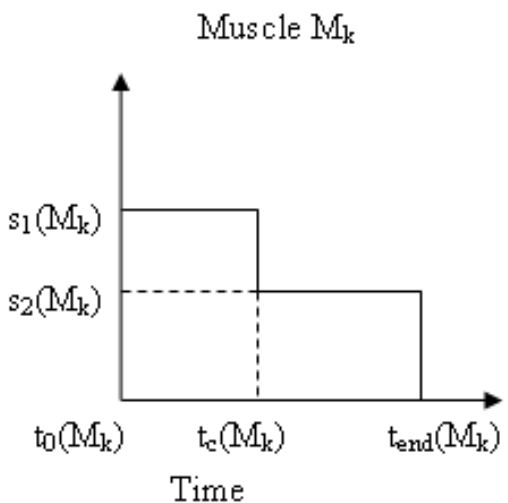

Figure 2: Stimulation profile for each muscle $M_{k} . t_{0}\left(M_{k}\right)$ is the starting time for the stimulation and $t_{\text {end }}\left(M_{k}\right)$ is the end time of the stimulation. $t_{c}\left(M_{k}\right)$ is the time at which the stimulation for muscle $M_{k}$ changes from $s_{1}\left(M_{k}\right)$ to $s_{2}\left(M_{k}\right)$.

problem. ${ }^{1}$ We strive to adjust the muscle stimulation timings for the leg movement, so that when the foot hits the ball, its obtained velocity is as high as possible. Considering the complexity and non-linearity of this problem, we decided to tackle it by using two evolution-based optimization methods: Particle Swarm Optimization (PSO) and Evolution Strategies (ES), which we describe in the following two sections.

Only the movement of the kicking leg was simulated. Movement of the pelvis was prescribed based on kinematic measurements of a soccer kick from a high level player. By defining the movement of the pelvis, it was not necessary to include the support leg in the simulation. The three dimensional coordinates of the ball relative to the body were allowed to change slightly. This allowed the body to reposition itself in relation to the ball to kick as fast as possible.

\section{Particle Swarm Optimization}

Particle Swarm Optimization (PSO) [12][11] is a relatively new optimization technique that is useful for parameter optimization in continuous and multi-dimensional search spaces. The original version of PSO was inspired by a model of bird flocking behavior [8] where the 'individuals' could fly over the solution space and land on the best solution. In PSO, search is performed by a 'population' of individuals $i$ with location vector $\overrightarrow{p_{i}}=\left(\overrightarrow{p_{i 1}}, \ldots, \overrightarrow{p_{i N}}\right)$, which represents a potential solution to the optimization problem. We can calculate the relative fitness of each individual $i$ by applying a fitness function $f$. Hence the fitness of an individual $i$ is defined by $f\left(\overrightarrow{p_{i}}\right)$. The fitter an individual, the more followers it will have. Along with its location $\overrightarrow{p_{i}}$, each individual keeps track of its 'velocity' $\overrightarrow{v_{i}}$. The velocity determines in which direction an individual will move and how far an individual will move in the next time step. The overall population moves through the problem space in

${ }^{1} 17$ muscles $* 3+2$ parameters for $M_{10}+3$ ball $(\mathrm{x}, \mathrm{y}, \mathrm{z})$ co-ordinates 


\begin{tabular}{|c|c|}
\hline Particle Swarm Optimization & Evolution Strategies \\
\hline 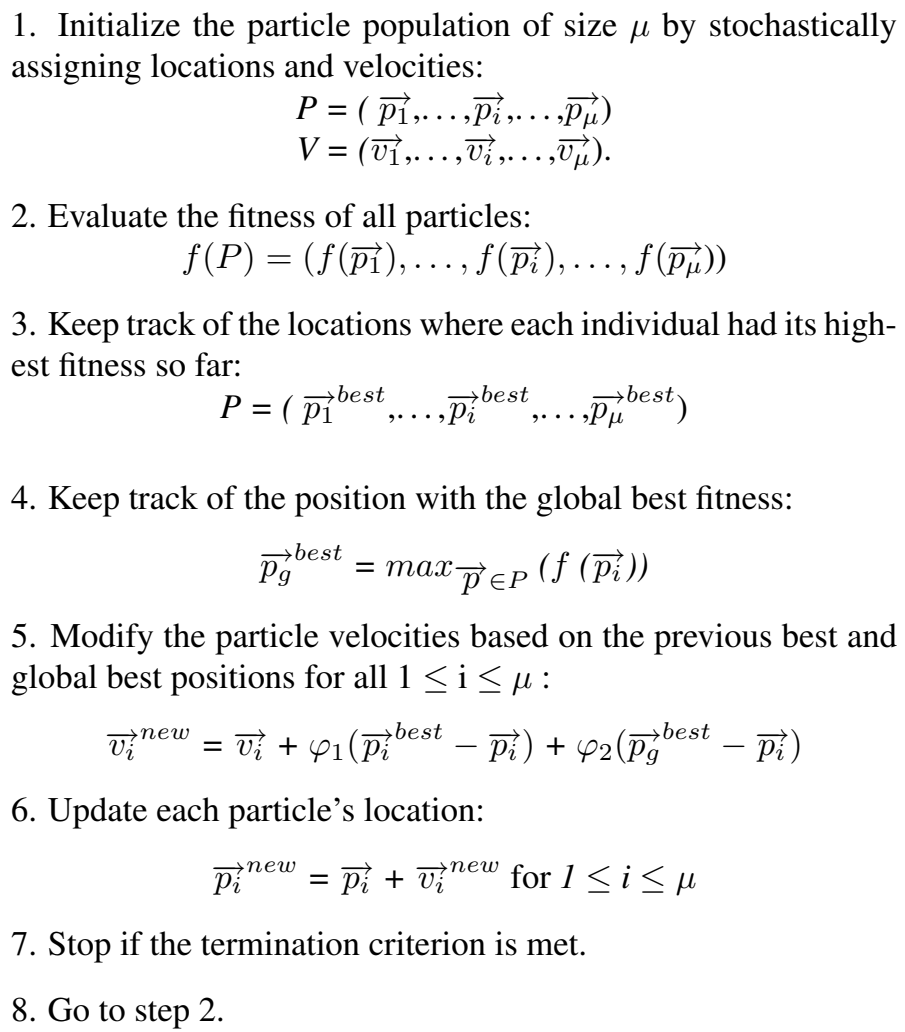 & $\begin{array}{l}\text { 1. Initialize the particle population with a single individual: } \\
\qquad P=\left(\overrightarrow{p_{0}}\right)=\left(\left(\overrightarrow{p_{01}}\right), \ldots,\left(\overrightarrow{p_{0 n}}\right)\right) \\
\text { 2. Calculate the fitness of the population: } f(P) \\
\text { 3. Find the individual with best fitness: } \\
\qquad \overrightarrow{p_{0}}=p \in P \text { such that } f(p)=\operatorname{Min}(f(P)) \\
\text { 4. Mutate } \overrightarrow{p_{0}} \text { : } \\
\text { a. Select a random direction, } \vec{r} \text { with a Gaussian random distri- } \\
\text { bution of } \sigma_{1}=\sigma_{2}=\ldots=\sigma_{n}=1 . \\
\text { b. Calculate a 5-point Lagrangian interpolation polynomial } \\
\text { (Ln }(x)) \text { of the restriction of } f, \text { centered at } p_{0} \text { and equidistant } \\
\text { with distance } h \text { for the points: } \overrightarrow{x_{0}}-2 h \vec{r}, \overrightarrow{x_{0}}-h \vec{r}, \overrightarrow{x_{0}}, \overrightarrow{x_{0}}+h \vec{r} \text {, } \\
\overrightarrow{x_{0}}+2 h \vec{r} . \\
\text { c. Find roots of the first derivative of the polynomial: } \\
\qquad L_{n}^{\prime}(x)=\frac{\partial L_{n}(x)}{\partial x}=0 . \\
\text { d. If there is a single root, } \lambda_{0}, P \text { is updated as follows: } \\
\qquad P=\left(\overrightarrow{p_{0}}, \overrightarrow{p_{0}} \lambda_{0} \vec{r}\right) \\
\text { e. If there are } 3 \text { roots, } \lambda_{1}, \lambda_{2}, \lambda_{3}, \text { then P is: } \\
\qquad P=\left(\overrightarrow{p_{0}}, \overrightarrow{p_{0}}+\lambda_{1} \vec{r}, \overrightarrow{p_{0}}+\lambda_{2} \vec{r}, \overrightarrow{p_{0}}+\lambda_{3} \overrightarrow{r^{\prime}}\right) \\
\text { 5. Go to step } 2 .\end{array}$ \\
\hline
\end{tabular}

(a)

(b)

Table 2: This table shows the PSO and ES algorithms used for the comparative study.

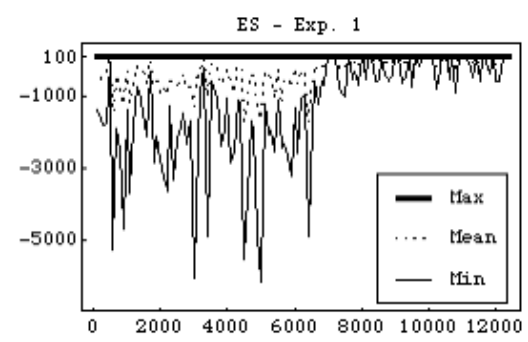

(a)

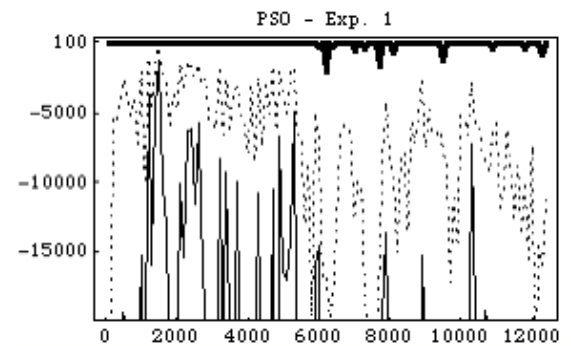

(b)

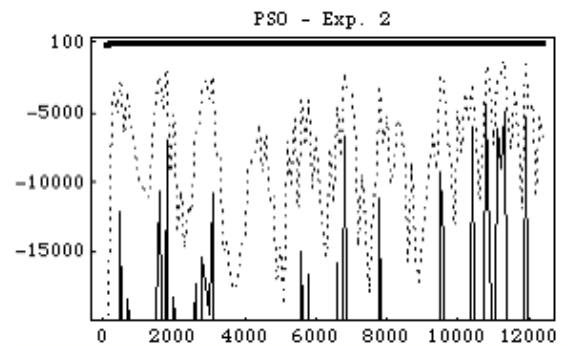

(c)

Figure 3: Fitness plot for each 100th iteration: The top curve (dark solid line) represents the maximum fitness at a particular iteration, the middle curve (dotted line) is the average fitness and the bottom curve (thin line) is the minimum fitness. (a) PSO experiment 1; (b) ES experiment 1 where the same start condition was used for PSO experiment 1; (c) PSO experiment 2; (Notice the scale of (a) is different.) 


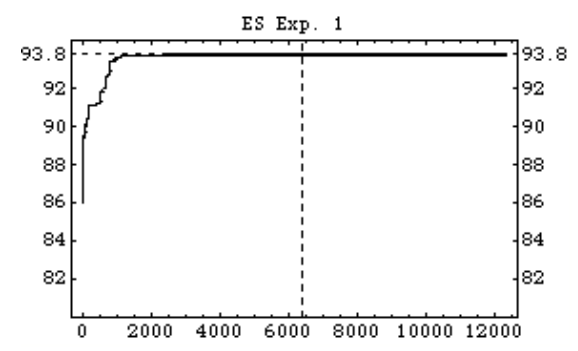

(a)

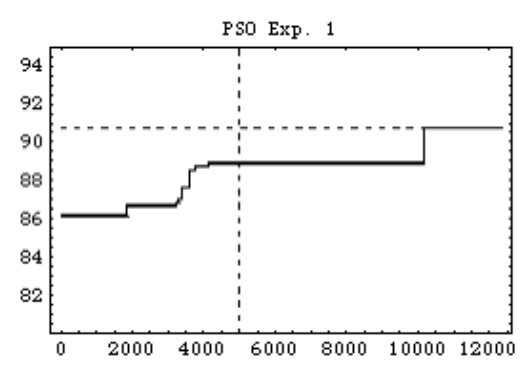

(b)

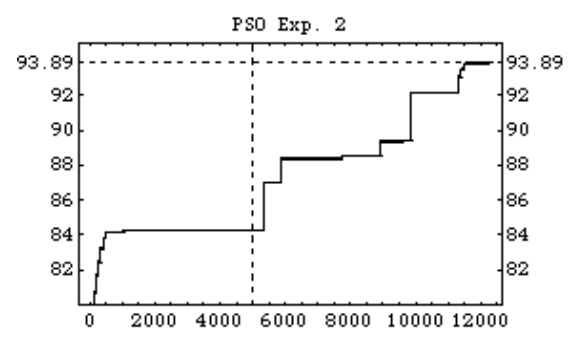

(c)

Figure 4: Best fitness achieved so far: The vertical line represents the iteration where our parameters were switched. (a) ES experiment 1 (b) PSO experiment 1 (c) PSO experiment 2

discrete time steps. The scheme of the Particle Swarm Optimizer we used for our experiments is outlined in Table 2(a).

Each PSO location vector $\overrightarrow{p_{i}}$ has the form:

$$
c t r l_{\text {sim,pso }}=\left(\operatorname{ctrl}_{M_{1}}, \ldots c t r l_{M_{17}}, \text { pos }_{\text {ball }}\right) .
$$

The fitness $f\left({ } t r l_{\text {sim,pso }}\right)$ is represented by:

$$
f\left(\operatorname{ctrl}_{\text {sim,pso }}\right)=\max _{\text {ball }}-\sum_{i=1}^{3} \text { contact }_{i}+\text { const }_{\text {toe }}
$$

where $\max _{\text {ball }}$ is the maximum forward ball speed and contact is a vector describing entries for three contact spheres that approximate the foot. If contact with all spheres occurs, the entry is zero, otherwise it is assigned a large value of 5,000 to ensure a high penalty for not touching the ball. The term const $_{\text {toe }}$ compares the end of each toe with a constraint position and is calculated as follows:

$$
\begin{gathered}
\text { const }_{\text {toe }}=\operatorname{Min}\left(\text { tiptoe }- \text { const }_{\text {pos }}, 0\right) \leq 0 \\
\text { const }_{\text {pos }}=z_{\text {ball }}-\text { radius }- \text { allow },
\end{gathered}
$$

where $z_{\text {ball }}$ is the vertical position of the ball. When the radius of the ball is subtracted one ends up at the ground level. Allow determines the lowest point the toes are allowed to go to. Otherwise, the toes would either be scrubbing the ground or actually kicking into the ground, none of which is acceptable. For our experiments allow was set to 2 $\mathrm{cm}$, which means that the model was constrained to prevent the toes from coming any closer than $2 \mathrm{~cm}$ vertically from the ground.

\section{Evolution Strategies}

Evolution Strategies (ES) have been developed since the 1960 's as a technique of evolutionary experimentation for solving complex optimization problems [10]. The preferred data structure are vectors of real numbers. Information between two vectors is interchanged by recombination operators. A slight variation on the vector elements is achieved by adding random values to each component (Table 2(b), Step 4).

The original ES experiments were performed in the $\mathrm{Hu}$ man Performance Lab (HPL), Faculty of Kinesiology in

\begin{tabular}{|l|l|l|}
\hline PSO Parameters & $\begin{array}{l}\text { Iteration } \\
0-5000\end{array}$ & $\begin{array}{l}\text { Iteration } \\
5001-12,330\end{array}$ \\
\hline \hline population size, $\mu$ & 10 & 10 \\
\hline \hline $\begin{array}{l}\text { velocity range, } v_{i j} \in \\
{\left[v_{\text {low }}, v_{\text {high }}\right]}\end{array}$ & {$[-0.1,0.1]$} & {$[-0.1,0.1]$} \\
\hline \hline $\begin{array}{l}\text { location range, } p_{i j} \in \\
{\left[p_{\text {low }}, p_{\text {high }}\right]}\end{array}$ & {$[0,1]$} & {$[0,1]$} \\
\hline \hline exploitation rate, $\varphi_{1}$ & 0.2 & 0.02 \\
\hline \hline exploration rate, $\varphi_{2}$ & 0.02 & 0.2 \\
\hline
\end{tabular}

Table 3: Parameter settings of the PSO for the Soccer Kick Simulation. (Table 2(a))

2002 [5][6]. This initially conducted experiment used a simple $(\mu+\lambda)$ ES scheme, for $\mu=1$, where a single parent $\overrightarrow{p_{0}}$ is mutated $\lambda$ times. Each of the offsprings is evaluated and the best offspring is selected as the parent for the next generation. The ES algorithm used for our experiment is outlined in Table 2(b).

For the ES individuals we expand on the PSO representation (Equation 2), however the ES strategy was also used to test the effect of adding mass in different regions of the shoe. Consequently, there are two extra parameters to take into account: the location where the extra mass is added $\left(\right.$ mass $\left._{l o c}\right)$ and the amount of mass $\left(\right.$ mass $\left._{a m t}\right)$. Hence, each ES individual $\overrightarrow{p_{i}}$ is represented as an expanded 58dimensional vector:

$$
c t r l_{\text {sim,es }}=\left(\operatorname{ctrl}_{\text {sim }, p s o}, \text { mass }_{l o c}, \text { mass }_{\text {amt }}\right) .
$$

The fitness of an ES individual $f\left(p_{i}\right)$, is calculated in the same way as for PSO, i.e., $f\left(c t r l_{\text {sim,es }}\right)=f\left(c t r l_{\text {sim,pso }}\right)$, described in Equation 3.

\section{Experiment Setup}

The population size for the PSO experiment is 10. The parameter settings used for our PSO experiment are listed in Table 3. We started off with an exploitation rate of 0.2 and an exploration rate of 0.02 . The exploration and exploitation values were switched after 5000 iterations.

The ES experiment started with an initial mutation radius of 0.01 and after approximately 6400 iterations this value was changed to 0.001 . The parameter settings are listed in 


\begin{tabular}{|l|l|l|}
\hline ES Parameters & $\begin{array}{l}\text { Iteration } \\
0-6400\end{array}$ & $\begin{array}{l}\text { Iteration } \\
6401-12,330\end{array}$ \\
\hline \hline population size & 2 or 4 & 2 or 4 \\
\hline \hline mutation radius, $h$ & 0.01 & 0.001 \\
\hline \hline$\lambda$ & 1 or 3 & 1 or 3 \\
\hline
\end{tabular}

Table 4: Parameter settings of the $(1+\lambda)$ ES for the Soccer Kick Simulation. (Table 2(b))

Table 4.

Various experiments were conducted with ES and PSO over the last year. We present our three best experimental results in the section below.

\section{Results}

The 3-D soccer kick model used in this simulation was developed using the DADS package, a software for dynamic motion simulation (Version 9.5) [1]. The model consists of rigid bodies representing a human right leg. The model was used to approximate real human movements by optimizing the muscle stimulations. The motion of the model is produced by following the stimulation schedule derived from ctrl $_{\text {sim,pso }}\left(\right.$ Equation 2) and $c t r l_{\text {sim,es }}$ (Equation 6), respectively.

The ES and PSO simulations were run for approximately 12,000 iterations $^{2}$ for the three experiments, each of which are described in the following subsections.

\subsection{Evolution Strategies - Experiment 1}

Our first experiment was conducted using ES. We started with random values for the 58-dimensional vector, ctrl $_{\text {sim,es. }}$. This initial configuration is visualized in Figure 5(a), where each set of muscle parameters is represented by three bars. The first bar is $t_{0}\left(M_{k}\right)$, the starting point of the stimulation for a particular muscle $M_{k}$, the second represents $t_{c}\left(M_{k}\right)$, the time when a muscle changes its stimulation, and the third bar is the end point of the stimulation $\left(t_{\text {end }}\left(M_{k}\right)\right)$. An exception is $M_{10}$ which has 5 bars defined by the parameters explained in Equation 2.

The initial configuration happens to have a high ball speed of $86.2 \mathrm{~km} / \mathrm{h}$. As seen in Figure 4(a), the fitness increases steadily for the first 1,500 generations. However, the fitness curve forms a plateau for another 10,000 generations. The ball speed of $93.72 \mathrm{~km} / \mathrm{h}$ is achieved in iteration 1117 , whereas the final best individual with a ball speed of $93.802 \mathrm{~km} / \mathrm{h}$ is obtained in iteration 6386 . Although the ball speed is high, it has a minor penalty associated with it (const $_{t o e}=1.9827$ ). The term, const $_{t o e}$ states that the foot should be at least $2 \mathrm{~cm}$ above the ground. Having a non-zero value, means that the foot was only $1.9827 \mathrm{~cm}$ above the ground, resulting in a minor penalty. When evaluating this

\footnotetext{
${ }^{2}$ So far we have only a few runs to compare our results with. The main reason is that the soccer kick simulation is time-consuming. To run a single-kick simulation, takes around 7 seconds. Given 10 individuals, evaluating a population takes 70 seconds. Running 12,330 iterations takes about 240 hours or 10 days.
}

solution against the PSO solution, we have chosen to ignore this penalty because the amount of the penalty $(0.2 \mathrm{~mm})$ is functionally negligible.

Figure 5(c) shows the difference between the first individual and the final best individual. We notice that the differences are small. A minor difference (around 0.05) is observed at the time when muscles $M_{3}$ and $M_{10}$ change their stimulation. Another minor difference occurs for $M_{15}$, which is responsible for knee extension. For the final best generation, the time when $M_{3}$ and $M_{10}$ changes stimulation is reduced compared to the first generation. This is why there is a minor penalty, as the inverted and extended foot along with the hip flexing is slightly deferred compared to the first iteration.

We notice in Figure 3(a) that ES individuals maintain a relatively low diversity. The fitness values between the best and the worst individuals at each generation do not change significantly. Figure 4(a), illustrates that the fitness rises significantly at the beginning and eventually ends up in a plateau.

\subsection{Particle Swarm Optimization - Experiment 1}

We conducted an experiment with PSO using the same initial individual as in the ES experiment (Section 6.1) (i.e., $c t r l_{\text {sim,es }}=\left(t r l_{\text {sim,pso }}\right)$. However, we set mass $_{l o c}=0$ and mass $_{a m t}=0$ since these do not take part in the evolution. Again, the final fitness value $f\left(c t r l_{\text {sim,pso }}\right)$ is relatively high. Figure 4(b) shows the fitness rising steadily until it reaches the value of $88.9 \mathrm{~km} / \mathrm{h}$. The fitness remains at this value for about 6,000 generations and finally reaches the value of $90.7 \mathrm{~km} / \mathrm{h}$ after 11,500 iterations. The evolved values for ctrl $_{\text {sim, pso }}$ are depicted in Figure 5(e). Compared to the best ES solution (Section 6.1), this individual does not have a penalty associated with it.

Figure $3 \mathrm{~b}$ shows the maximum, minimum and average fitnesses at every 100th iteration. Comparing the two fitness curves, Figure 3(a) and 3(b), we observe that the diversity of the PSO individual is high during the whole evolution experiment.

\subsection{Particle Swarm Optimization - Experiment 2}

In order to see whether PSO is competitive to ES, we conducted another PSO experiment, where we began with a different set of initial conditions that resulted in a high penalty, hence giving a fitness value of -90 (Figure 4(c), not shown in the graph). The term const toe has a penalty where the toe goes below the virtual ground and can be seen in Figure 6. After the first 5000 iterations the simulation seems to have reached a fitness plateau (see the vertical line in Figure 4(c)). At this point we interchanged the exploration and exploitation rates as outlined in Table 3 .

After iteration 12,248 we obtained a fitness value of $93.89 \mathrm{~km} / \mathrm{h}$ (Figure 7). The speed reported for an average skilled player is $91 \mathrm{~km} / \mathrm{h}$ [3]. We also observed that the foot touches the ball for about $7 \mathrm{~ms}$, close to results of $9 \mathrm{~ms}$ reported by Asai and coworkers [2] using high speed video techniques. 


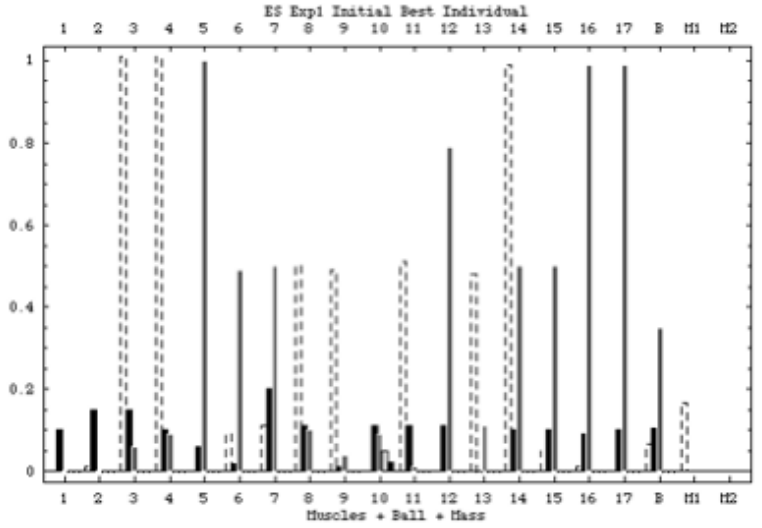

(a)

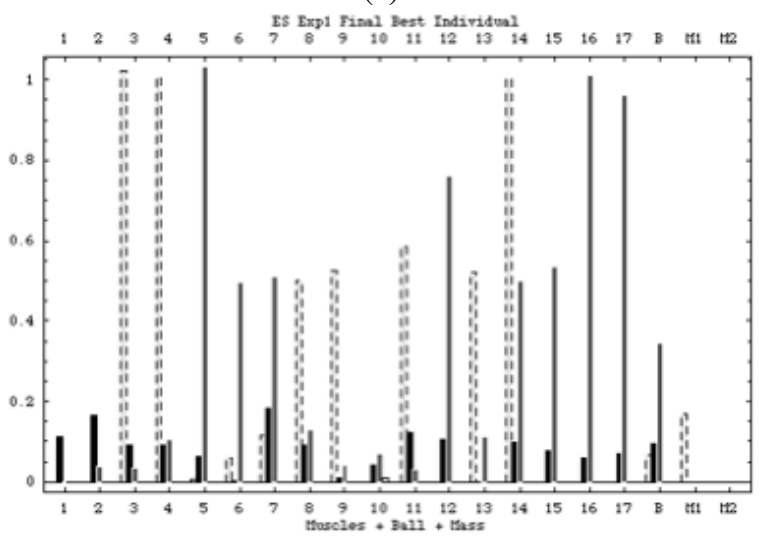

(b)

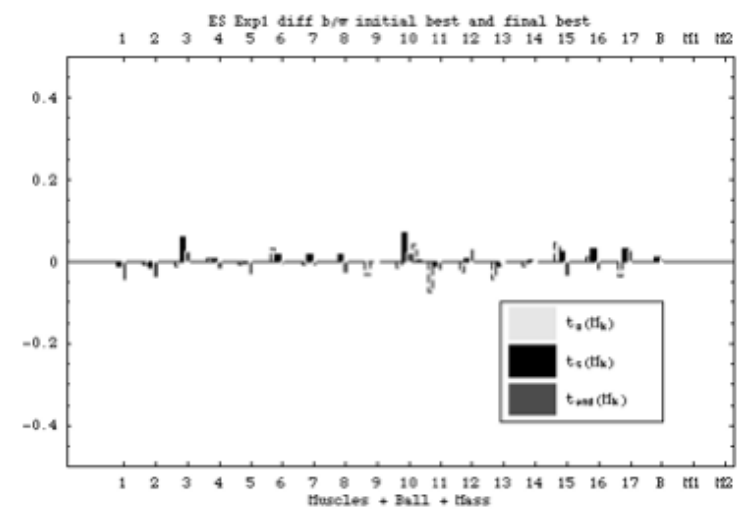

(c)

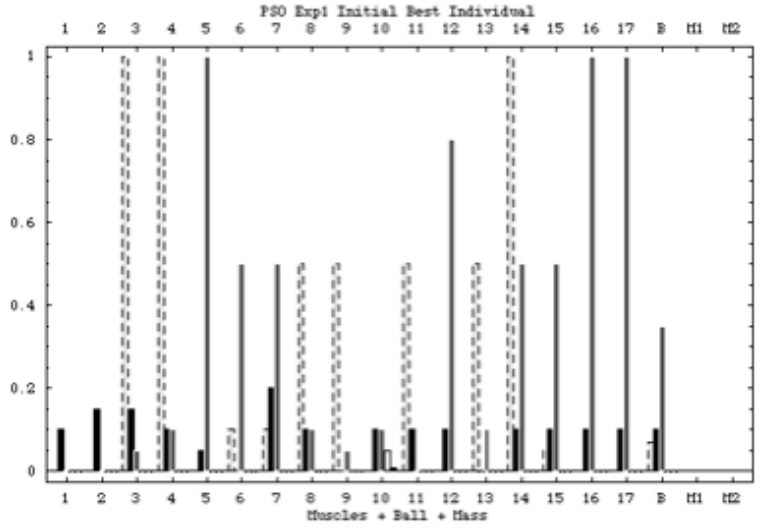

(d)

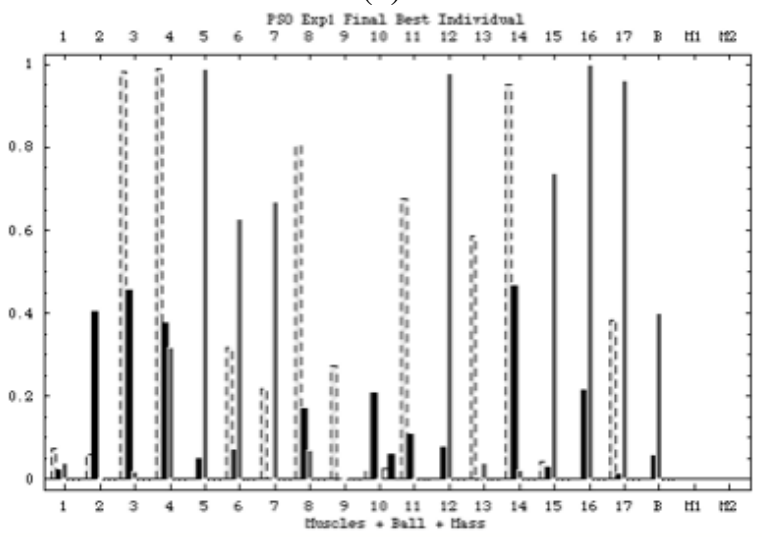

(e)

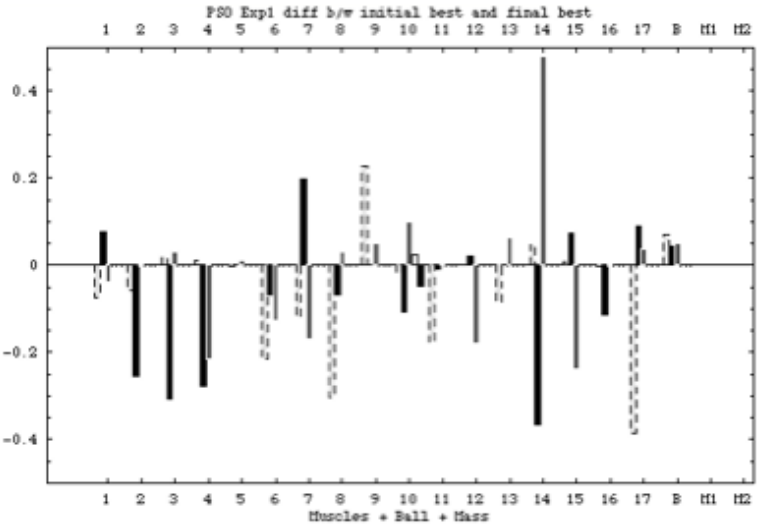

(f)

Figure 5: ES and PSO experiments with the same initial start configuration: (a) ES individual at iteration 1; (b) ES best individual at iteration 6,356; (c) ES difference between (a) and (b); (d) PSO individual at iteration 1; (e) PSO best individual at iteration 4,123; (f) PSO difference between (d) and (f).
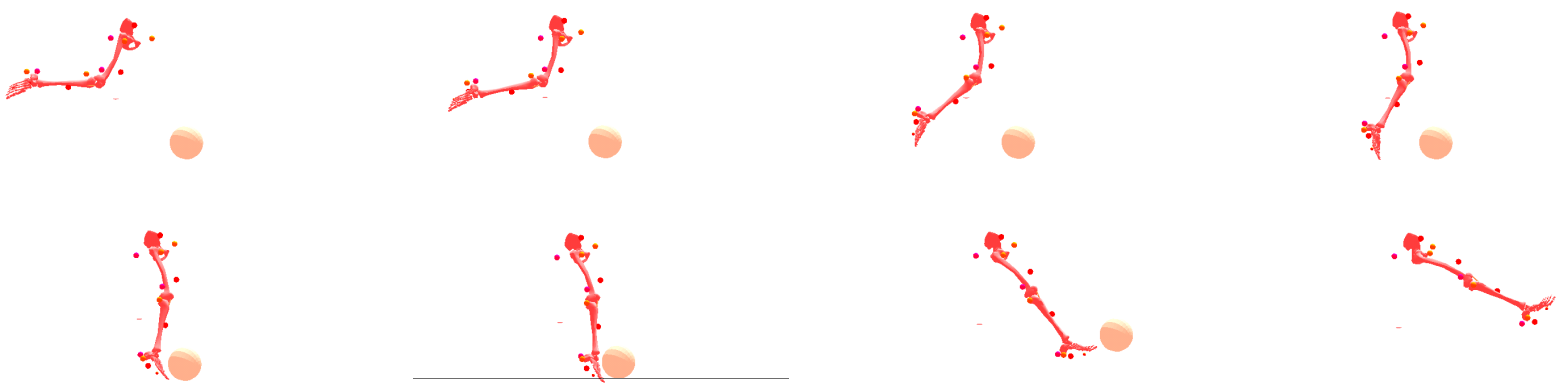

Figure 6: Leg movement from the initial best solution in iteration 1 using PSO (Experiment 2). Notice that the toes go below the virtual floor resulting in a penalty. 

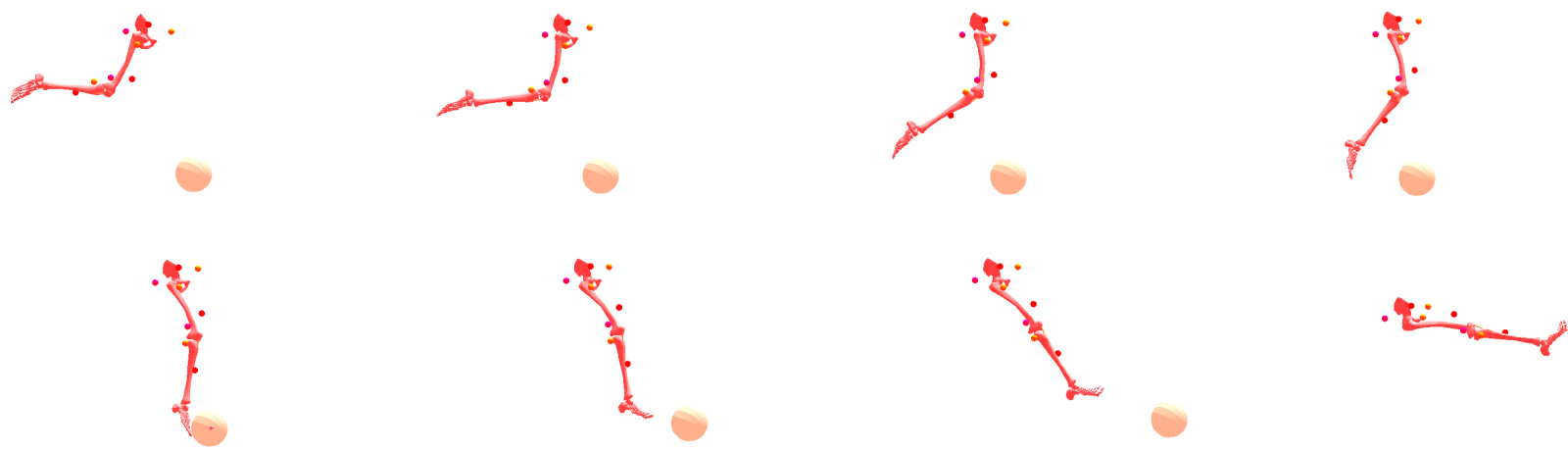

Figure 7: Leg movement resulting from the final best solution found in iteration 12,248 using PSO (Experiment 2).

Figure 8 compares the best individual in the first iteration with the individual with the best fitness value (ball speed) of 93.89 after 12,330 iterations. Since we obtained a good solution for the PSO individual, we explain the details of the final ctrl $_{\text {sim,pso. }}$ Figures 8(a) and 8(b) show which of the muscles are activated and deactivated at particular points in time during the kicking movement. The human leg acts as a double pendulum as illustrated in Figure 6; one joint is at the hip and the other is at the knee. The main muscle affecting the movement of the hip is $M_{3}$ (Iliopsoas). Inititally this muscle is highly activated to produce the hip movement during the early stages of the kick (Figure 6). When the hip has completed its action, the muscle deactivates. Once the hip movement is completed, the movement from the knee contributes mostly to the kick. Muscles affecting the movement of the knee are: $M_{5}$ (Vastus Itermedius), $M_{15}$ (Vastus Medialis), $M_{16}$ (Vastus Lateralis) and $M_{17}$ (Castus Medialis Oblique). These four muscles combine to form the quadriceps. Initially none of these muscles are stimulated. Only after the hip movement is completed, they are strongly activated. Muscles $M_{6}$ (Gastrocnemius) and $M_{7}$ (Soleus) causes the foot to go back in order to get the foot to contact the ball. These two muscles are highly activated towards the end. $M_{10}$ (Tibialis Anterior) remains activated, however $M_{12}$ (Extension Digitorum) along with $M_{10}$ deals with the stiffness of the ankle. This stiffening is important for a good kick.

\section{Discussion and Future Work}

In this paper we have described our implementation of the soccer kick project, along with our initial experiments performed with both evolution strategies and the particle swarm optimizer. We used these evolutionary algorithms to adjust the muscle control parameters for a soccer kick.

It is well recognized within the biomechanics community that the system of muscles in the lower extremity is over-determined for the number of degrees of freedom available for movement. Multiple solutions for muscle recruitment patterns are possible. The only means available to further validate the solutions obtained via numerical optimization is to qualitatively compare them against experimental measurements of the muscle activation patterns. From this perspective, both ES and PSO determined solu-

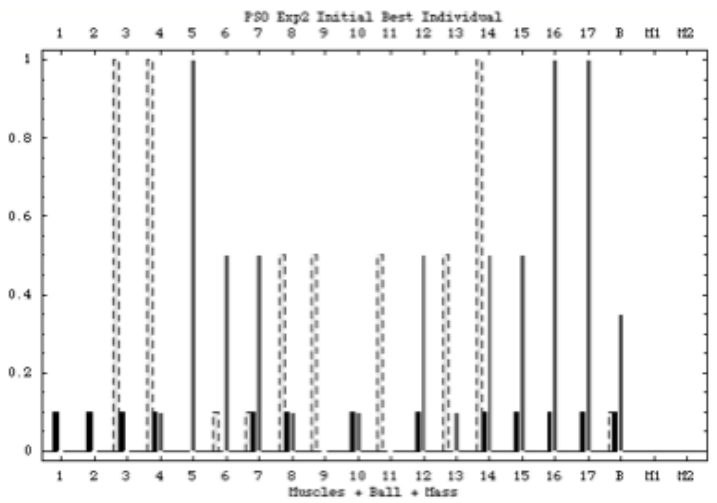

(a)

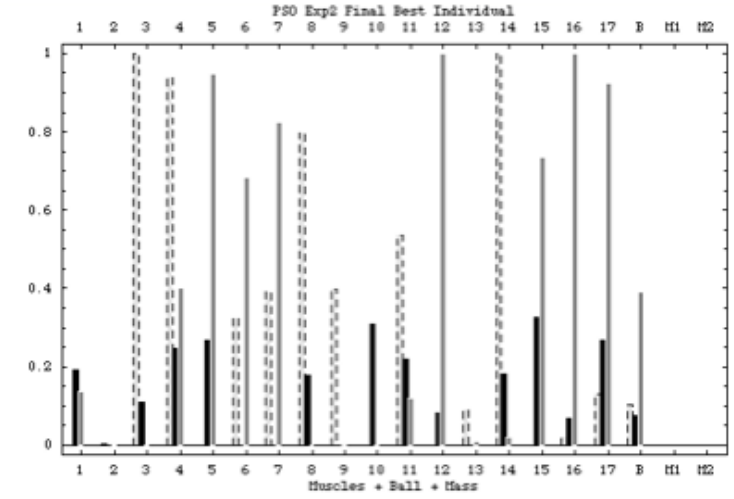

(a)

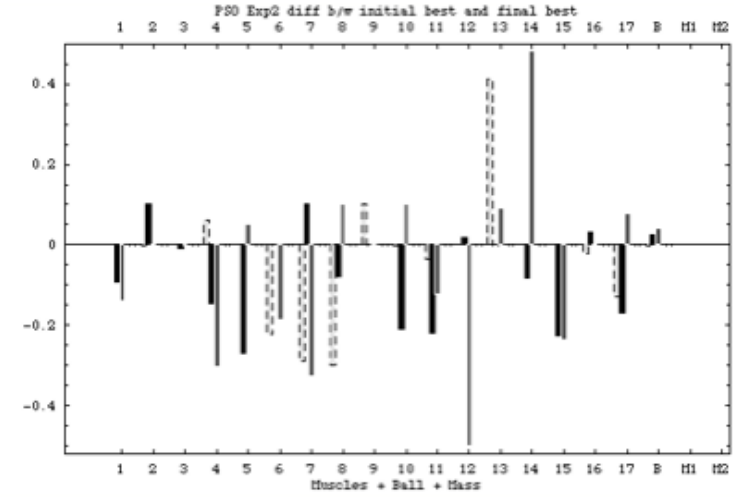

(c)

Figure 8: PSO experiment 2 (a) Best individual at iteration 1; (b) Final best individual at iteration 12,248; (c) Difference between (a) and (b). 
tions to the soccer kick problem that were biomechanically reasonable. Further discussion of these solutions from a biomechanical perspective is beyond the scope of this paper.

Given the nature of the biomechanical problem presented in this paper, the numerical optimization algorithms need to be evaluated on three different levels:

- Do the algorithms reach a good estimate of the maximum fitness? This is particularly relevant when using the model to optimize athletic equipment design, and the sensitivity of the optimal solution needs to be understood.

- How many iterations are required to reach a good estimate of the maximum fitness?

- Can the algorithm provide information about multiple solutions? This is of interest when using the model to study aspects of neuro-muscular control of human movement.

In order to answer the above questions, we need to perform more experiments. Based on the results thusfar, ES performs a little better for this problem than PSO; ES reached a good solution with fewer iterations and for the same initial configuration ES had a slightly higher ball velocity (ES Experiment 1 - Section 6.1 and PSO Experiment 1 - Section 6.2). However, ES was set up as a simple local search algorithm- $(1+\lambda)$ scheme-and the initial starting point gave a decent kick. As of yet we do not know how ES would behave from a starting point with an unreasonable kick (PSO Experiment 2 - Section 6.3).

So far, the control parameters for the ES and PSO algorithms were tuned manually, which basically leads to a trial and error approach. This is not only time consuming but does not guarantee optimal results at all. Consequently, we will strive to automate the procedure of optimizing these control parameters, which will lead to a hierarchical metaoptimizer.

Different variants of PSO exist. We will investigate the influence of inertia weight [15][16], constriction factor [4] and the scaling terms $\left(\varphi_{1}, \varphi_{2}\right)$ on optimization algorithm performance. A recent version of the PSO algorithm incorporating dynamic inertia and velocity updating will also be evaluated [9]. This algorithm has been used in recent work [14] and has shown promising results.

\section{Bibliography}

[1] Lms international - www.lmsintl.com.

[2] T. Asai and M. Kaga. Impact process of kicking in football. Proceedings of the XVth Congress of the International Society of Biomechanics, 1995.

[3] W. Barfield and D. Kirkendall. Journal of sports science and medicine. 1:72-79, 2002.

[4] M. Clerc. The swarm and the queen: towards a deterministic and adaptive particle swarm optimization.
Proceedings of the IEEE Congress on Evolutionary Computation (CEC 1999), 1999.

[5] G. Cole and K. Gerritsen. Influence of mass distribution in the shoe and plate stiffness on ball velocity during a soccer kick. Adidas-Salomon AG, 2002.

[6] G. Cole and K. Gerritsen. Optimal mass distribution and plate stiffness of football shoes:part2. AdidasSalomon AG, 2002.

[7] G. Cole and K. Gerritsen. Influence of medio-lateral mass distribution in a soccer shoe on the deflection of the ankle and subtalar joints during "off-centre" kicks. Adidas-Salomon AG, 2003.

[8] R. C. Eberhart, J. Kennedy, and Y. Shi. Swarm Intelligence: Collective Adaptation. Morgan Kaufmann, San Francisco, 2000.

[9] P. Fourie and A. Groenwold. The particle swarm optimization in size and shape optimization. Structural and Multidisciplinary Optimization, 23:259267, 2002.

[10] C. Jacob. Illustrating Evolutionary Computation with Mathematica. Morgan Kaufmann Publishers, San Francisco, CA, 2001.

[11] C. Jacob and N. Khemka. Particle swarm optimization in mathematica an exploration kit for evolutionary optimization. In Proceedings of the Sixth International Mathematica Symposium, 2004.

[12] J. Kennedy and R. C. Eberhart. Swarm Intelligence. The Morgan Kaufmann Series in Evolutionary Computation. Morgan Kaufmann Publishers, San Francisco, 2001.

[13] I. Rechenberg. Evolution strategy. In J. M. Zurada, R. J. Marks II, and C. J. Robinson, editors, Computational Intelligence Imitating Life. IEEE Press, New York, 1994.

[14] B. Schutte and B. Fregly. Evaluation of a particle swarm algorithm for biomechanical optimization. Journal of Biomechanical Engineering, 127, June, 2005.

[15] Y. Shi and R. C. Eberhart. A modified particle swarm optimizer. Proceedings of the IEEE Congress on Evolutionary Computation (CEC 1998), 1998.

[16] Y. Shi and R. C. Eberhart. Particle swarm optimization with fuzzy adaptive inertia weight. Proceedings of the IEEE Congress on Evolutionary Computation (CEC 2001), 2001.

[17] I. Wright and K. Neptune. Passive regulation of impact forces in heel to toe running. Clinical Biomechanics, 13:521-531, 1998. 